\title{
High flow nasal catheter therapy versus non-invasive positive pressure ventilation in acute respiratory failure (RENOVATE trial): protocol and statistical analysis plan
}

Israel S Maia, Leticia Kawano-Dourado, Fernando G Zampieri, Lucas P Damiani, Renato H Nakagawa, Rodrigo M Gurgel, Karina Negrelli, Samara PC Gomes, Denise Paisani, Lucas M Lima, Eliana V Santucci, Nanci Valeis, Ligia N Laranjeira, Roger Lewis, Mark Fitzgerald, Carlos RR Carvalho, Laurent Brochard and Alexandre B Cavalcanti, for the RENOVATE

Investigators and the BRICNet

Up to $49 \%$ of intensive care unit (ICU) admissions are due to acute respiratory failure (ARF). ${ }^{1}$ Respiratory support may be provided through invasive or noninvasive ventilation. The incidence of ARF demanding invasive mechanical ventilation has been reported to be 137 hospitalisations per 100000 population older than 5 years of age, with $36-48 \%$ mortality. ${ }^{1,2}$ Avoiding unnecessary invasive mechanical ventilation is important because this type of respiratory support is not exempt from complications. ${ }^{3-6}$

High flow nasal catheter (HFNC) therapy and noninvasive positive pressure ventilation (NIPPV) are used for respiratory support in patients with ARF. HFNC therapy is a newer method of initial respiratory support which enables delivery of continuous high gas flow (up to $70 \mathrm{~L} / \mathrm{min}$ ) via nasal prongs, high fraction of inspired oxygen $\left(\mathrm{FiO}_{2}\right)$ and some positive end-expiratory pressure (PEEP). ${ }^{7,8}$ NIPPV has been used to avoid intubation at ICU arrival in $20 \%$ of ARF patients overall. ${ }^{9}$ By delivering positive pressure during respiration, NIPPV increases functional residual capacity and opens collapsed alveoli, which contributes to increasing ventilation and decreasing intrapulmonary shunting. Furthermore, it improves lung compliance, decreases the work of breathing, reduces afterload and increases cardiac output, so it contributes to improved oxygenation. ${ }^{10,11}$ HFNC therapy acts through different mechanisms. It improves oxygenation, providing stable alveolar $\mathrm{FiO}_{2}$; it increases carbon dioxide clearance, decreasing anatomic dead space; it reduces the work of breathing and the respiratory rate; and it increases pharyngeal pressure and lung volume, contributing to a PEEP effect (although less remarkable compared with NIPPV). Moreover, lung stress and strain are decreased, and patient comfort is improved, allowing patients to eat, drink and talk without therapy interruption. 8,12-17

Heterogeneity is also present in the pathophysiology of different aetiologies of ARF. For hypoxaemic ARF, acute lung injury promotes increased pulmonary

\section{ABSTRACT}

Background: The best way to offer non-invasive respiratory support across several aetiologies of acute respiratory failure (ARF) is presently unclear. Both high flow nasal catheter (HFNC) therapy and non-invasive positive pressure ventilation (NIPPV) may improve outcomes in critically ill patients by avoiding the need for invasive mechanical ventilation (IMV).

Objective: Describe the details of the protocol and statistical analysis plan designed to test whether HFNC therapy is noninferior or even superior to NIPPV in patients with ARF due to different aetiologies.

Methods: RENOVATE is a multicentre adaptive randomised controlled trial that is recruiting patients from adult emergency departments, wards and intensive care units (ICUs). It takes advantage of an adaptive Bayesian framework to assess the effectiveness of HFNC therapy versus NIPPV in four subgroups of ARF (hypoxaemic non-immunocompromised, hypoxaemic immunocompromised, chronic obstructive pulmonary disease exacerbations, and acute cardiogenic pulmonary oedema). The study will report the posterior probabilities of non-inferiority, superiority or futility for the comparison between HFNC therapy and NIPPV. The study assumes neutral priors and the final sample size is not fixed. The final sample size will be determined by a priori determined stopping rules for non-inferiority, superiority and futility for each subgroup or by reaching the maximum of 2000 patients.

Outcomes: The primary endpoint is endotracheal intubation or death within 7 days. Secondary outcomes are 28-day and 90-day mortality, and ICU-free and IMV-free days in the first 28 days.

Results and conclusions: RENOVATE is designed to provide evidence on whether HFNC therapy improves, compared with NIPPV, important patient-centred outcomes in different aetiologies of ARF. Here, we describe the rationale, design and status of the trial.

Trial registration: ClinicalTrials.gov NCT03643939.

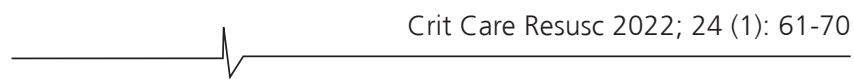


vascular permeability, increased lung weight, loss of aerated lung tissue, alveolar collapse and shunting. ${ }^{18,19}$ During acute exacerbations of chronic obstructive pulmonary disease (COPD), there are increases in the elastic and resistive loads on the respiratory muscles which may lead to ventilatory failure. ${ }^{20,21}$ In cardiac failure, increased back pressure on the pulmonary circulation leads to extravasation of fluid into the alveoli, hence lungs become less compliant, which increases the effort of breathing, shunting and ventilationperfusion mismatch. 22,23

Studies comparing HFNC therapy and NIPPV in specific aetiologies of ARF have been published. Hypoxaemic non-immunocompromised patients were evaluated in one large randomised controlled trial which showed no difference between HFNC therapy compared with NIPPV in endotracheal intubation rate at Day $28 .{ }^{17}$ In a recent network meta-analysis, no significant difference in endotracheal intubation was observed when comparing HFNC therapy versus face mask NIPPV, although the quality of evidence was low. ${ }^{24}$ Also, to our knowledge, no randomised controlled trials have compared HFNC therapy and NIPPV in immunocompromised patients other than two post-hoc analyses, of which only one showed benefit in the HFNC group. ${ }^{25-27}$ Trials in acute cardiogenic pulmonary oedema (ACPO) looking at endotracheal intubation risk or death are lacking. Few small trials of patients with acute COPD exacerbations have been reported, and no conclusions regarding benefit or harm in those receiving HFNC therapy have been made. ${ }^{28}$ Consequently, there is significant uncertainty regarding the efficacy of HFNC therapy versus NIPPV in reducing endotracheal intubation rate and death in these aetiologies of ARF.

RENOVATE was designed with an adaptive framework to compare use of a newer treatment technology - the HFNC - with NIPPV. In the trial, patients presenting with ARF will be categorised into one of four pre-specified groups:

- hypoxaemic non-immunocompromised;

- hypoxaemic immunocompromised;

- COPD exacerbations; and

- acute cardiogenic pulmonary oedema.

The primary endpoint for all groups is a binary response to whether intubation or death occurs within 7 days of treatment for ARF. Also, since HFNC therapy has clinical benefits that are unrelated to the intubation rate, such as better comfort for patients, we aim to determine both noninferiority and superiority of HFNC therapy versus NIPPV. This protocol explains how the Bayesian adaptive unique design ensures high quality methods and contributes to transparency in future reporting and analysis of this trial.

\section{Objectives and outcomes}

The primary objective of the trial is to determine noninferiority of HFNC therapy to NIPPV, in addition to testing for superiority. The primary outcome is endotracheal intubation or death in 7 days. Secondary outcomes are 28day and 90-day mortality, and ventilator-free days and ICUfree days truncated at 28 days. The primary, secondary and tertiary outcomes are shown in Table 1.

\section{Methods}

\section{Study design, randomisation and allocation concealment}

RENOVATE is an adaptive, open label, multicentre, noninferiority randomised controlled trial comparing HFNC therapy versus NIPPV in patients with ARF of different aetiologies. Patients with ARF are being randomly assigned $1: 1$ to receive HFNC therapy or NIPPV according to the flowchart shown in Figure 1.

The randomisation list is generated electronically in blocks using validated software. Allocation concealment is maintained via an online central automated system available 24 hours per day. The arm to which a patient is allocated is only disclosed after registration in the electronic system. The randomisation is stratified by centre and by ARF aetiology (Figure 1). Blinding is not possible because of the characteristics of the intervention, but outcome evaluators are blinded to treatment group allocation.

\section{Sites and recruitment status}

Patients are being recruited in adult emergency departments, wards and ICUs in 35 centres. The first patient was enrolled on 11 November 2019. The planned trial duration is estimated at 2.5 years.

\section{Table 1. Study endpoints}

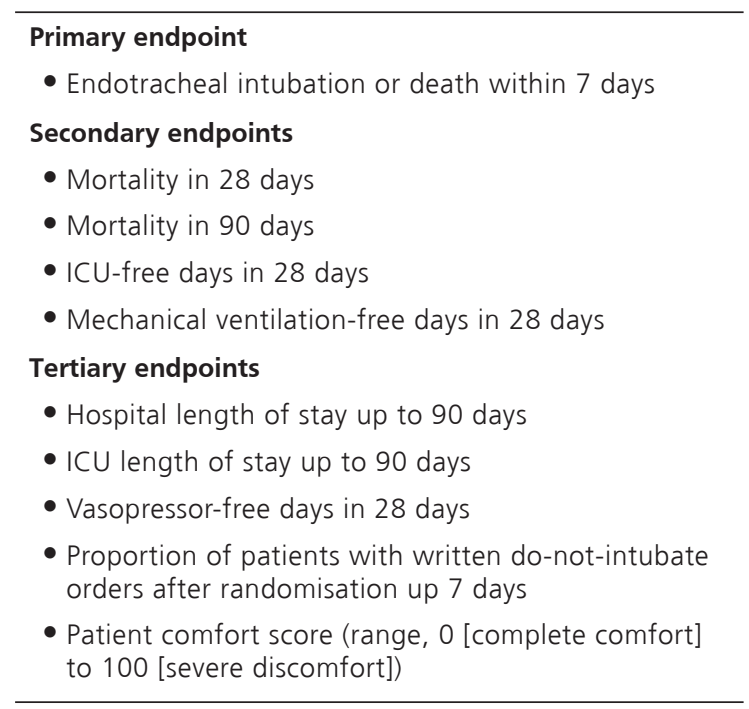

$\mathrm{ICU}=$ intensive care unit. 
Figure 1. RENOVATE study flowchart

Acute respiratory failure groups

\begin{tabular}{|c|c|c|}
\hline $\begin{array}{c}\text { Hypoxaemic } \\
\text { non-immunocompmised }\end{array}$ & $\begin{array}{c}\text { Hypoxaemic } \\
\text { immunocompromised }\end{array}$ & $\begin{array}{l}\text { Chronic obstructive } \\
\text { pulmonary disease }\end{array}$ \\
\hline
\end{tabular}

Web-based randomisation-ratio 1:1, stratified by cause of the acute respiratory failure (group) and centre

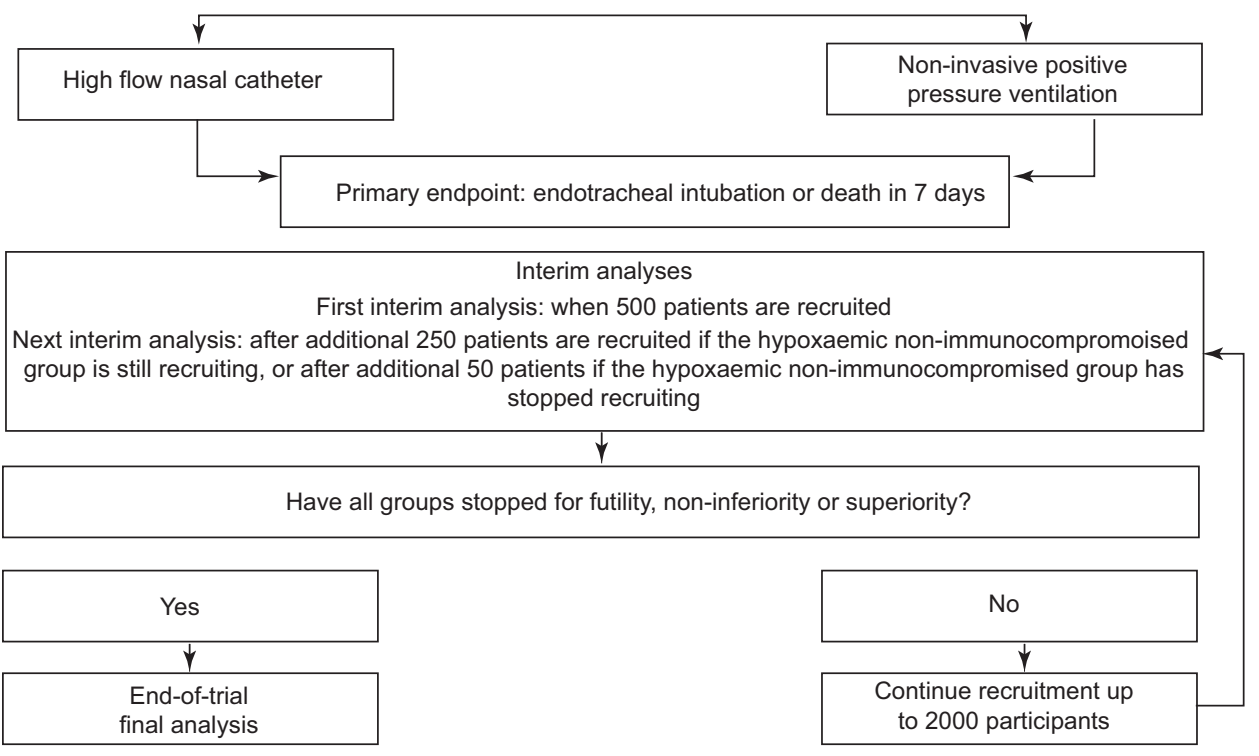

\section{Inclusion and exclusion criteria}

Patients must be $\geq 18$ years old, with respiratory discomfort of acute onset. Eligibility criteria for each ARF group are detailed in Table 2.

\section{Initial pre-randomisation approach}

Patients should receive the standard of care in the period between eligibility check and randomisation. The use of NIPPV in this period is allowed if it is for less than 6 hours. For ACPO patients, any use of NIPPV renders the patient ineligible. Centres will provide the standard of care for patients as needed in this period; if NIPPV is deemed necessary, it will not be withheld to include the patient in the study.

\section{Study interventions}

\section{Experimental arm - high flow nasal catheter therapy}

In the HFNC therapy arm, flow starts at $30 \mathrm{~L} / \mathrm{min}$ for COPD patients, and $45 \mathrm{~L} / \mathrm{min}$ for hypoxaemic ARF and ACPO patients, titrating gradually towards $60 \mathrm{~L} / \mathrm{min}$ or the highest flow tolerated. ${ }^{12}$ The temperature of the humidified circuit is maintained at $34-37^{\circ} \mathrm{C}$, and the $\mathrm{FiO}_{2}$ is titrated to maintain peripheral oxygen saturation at $92-98 \%$ in all patient groups except COPD patients, for whom it should be maintained at 88-92\%. After 24 hours, weaning from HFNC therapy may begin if there is clinical improvement starting with $\mathrm{Fio}_{2}$ followed by flow as tolerated by the patient. Patients are considered weaned whenever $30 \%$ $\mathrm{FiO}_{2}$ and $\leq 25-30 \mathrm{~L} / \mathrm{min}$ of flow are achieved while $\mathrm{SpO}_{2}$ is maintained within target range. Rescue with NIPPV is allowed only in scenarios where there is clear evidence of NIPPV benefit: COPD and ACPO ${ }^{30}$ (Figure 2).

\section{Control arm, standard treatment - non-invasive positive pressure ventilation}

NIPPV is performed using oronasal or total face mask with ICU ventilator or bi-level devices with a single circuit and an exhalation valve according to availability at each centre. The ventilation mode should be pressure support (PS), tidal volume target set at 6-9 mL/kg of ideal body weight ${ }^{31}$ and proper synchrony. After 24 hours, weaning from NIPPV may begin if there is clinical improvement. Duration of each NIPPV session, $\mathrm{FiO}_{2}$ and pressures are decreased progressively each day at clinical team discretion. The patient is considered weaned whenever $30 \% \mathrm{FiO}_{2}$, expiratory positive airway pressure and pressure support $\leq 6 \mathrm{cmH}_{2} \mathrm{O}$ are achieved while $\mathrm{Spo}_{2}$ is maintained within target range. 
Table 2. Inclusion and exclusion criteria

\section{Inclusion criteria}

Aged 18 years or older, admitted to intensive care unit or emergency department with acute respiratory discomfort, and belonging to one of the following groups (with their respective criteria)

\section{Hypoxaemic non-immunocompromised: criteria A plus either B and/or C}

A. Hypoxaemia evidenced by peripheral oxygen saturation $<90 \%$ or arterial partial pressure of oxygen $<60 \mathrm{mmHg}$ at room air

B. Use of accessory musculature, paradoxical breathing, and/ or thoraco-abdominal asynchrony

C. Respiratory rate $>25$ breaths/min

\section{Hypoxaemic immunocompromised: criteria A and B plus} either C and/or D

A. Immunosuppression diagnosis*

B. Hypoxaemia evidenced by peripheral oxygen saturation $<90 \%$ or arterial partial pressure of oxygen $<60 \mathrm{mmHg}$ at room air

C. Use of accessory musculature, paradoxical breathing, and/ or thoraco-abdominal asynchrony

D. Respiratory rate $>25$ breaths/min

\section{COPD exacerbation: Criteria A, B and C}

A. High clinical suspicion of COPD or previous COPD diagnosis $^{\dagger}$

B. Respiratory rate $>25$ breaths/min or use of accessory musculature, paradoxical breathing and or thoraco-abdominal asynchrony

C. Arterial blood $\mathrm{pH}<7.35$ and arterial partial pressure of carbon dioxide $>45 \mathrm{mmHg}$

\section{Acute cardiogenic pulmonary oedema: criteria A, B and C}

A. Acute cardiogenic pulmonary oedema diagnosis ${ }^{\ddagger}$

B. Respiratory rate $>25$ breaths/min

C. Peripheral oxygen saturation $<95 \%$

\section{Exclusion criteria}

- Indication of emergency endotracheal intubation:

- prolonged respiratory pauses

- cardiorespiratory arrest

- Glasgow coma scale score $\leq 12$

- heart rate $<50$ beats/min with decreased level of consciousness

- arterial blood $\mathrm{pH}<7.15$ irrespective of the cause

- Psychomotor agitation that prevents adequate medical/nursing care requiring heavy sedation

- Persistent haemodynamic instability with mean arterial pressure $<65 \mathrm{mmHg}$, systolic arterial pressure $<90 \mathrm{mmHg}$ after adequate volume resuscitation or requiring norepinephrine $>0.3 \mu \mathrm{g} / \mathrm{kg} / \mathrm{min}$ or equivalent

- Contraindications to non-invasive ventilation: face deformities or traumas, recent oesophageal surgery, hypersecretion, vomiting with aspiration risk

- Presence of pneumothorax or extensive pleural effusion

- Severe arrhythmia that puts patient at risk of haemodynamic instability

- Thoracic trauma understood to be the main cause of ARF

- Asthma attack

- Pregnancy

- Cardiogenic shock

- Acute coronary syndrome with plans to undergo coronary angiography within $24 \mathrm{~h}$

- ARF up to $72 \mathrm{~h}$ after orotracheal extubation

- Post-surgical ARF (surgery within 72 h)

- Hypercapnic ARF due to neuromuscular disease or chest deformities

- Patient is receiving exclusive palliative care

- Do-not-intubate order

- Chronic pulmonary disease except COPD

- Use of $>6$ h of NIPPV before randomisation in hypoxaemic nonimmunocompromised patients, hypoxaemic immunocompromised patients and patients with COPD exacerbation

- Use of NIPPV before randomisation in acute cardiogenic pulmonary oedema patients

ARF = acute respiratory failure. COPD = chronic obstructive pulmonary disease. NIPPV = non-invasive positive pressure ventilation. * Regular use of immunosuppressor drugs in the long term (> 3 months) or high doses of corticosteroids (> 0.5 mg/ $/ \mathrm{kg} / \mathrm{day}$ ); solid organ transplants; solid tumour with need for chemotherapy in the past 5 years; haematologic malignant diseases with treatment in the past 5 years; infection by HIV with AIDS primary immunodeficiency. ${ }^{29}$ + Smoker or former smoker, or exposure to the burning of biomass; chronic dyspnoea and/or chronic productive cough; exclusion of other causes of chronic respiratory symptoms (eg, pulmonary fibrosis, congestive cardiac failure, cystic fibrosis). $\neq$ Sudden-onset dyspnoea and diffuse crackling rales with or without the third audible heart sound; lack of history of pulmonary aspiration, infection or pulmonary fibrosis; acute cardiogenic pulmonary oedema as a main clinical hypothesis; chest x-ray with bilateral alveolar infiltrates suggestive of pulmonary oedema.

\section{Endotracheal intubation}

Endotracheal intubation indications are the same for both study arms and are shown in Table 3. The final decision regarding use of endotracheal intubation is made by the attending physician, who may or may not be a site investigator. 
Figure 2. Intervention failure algorithm
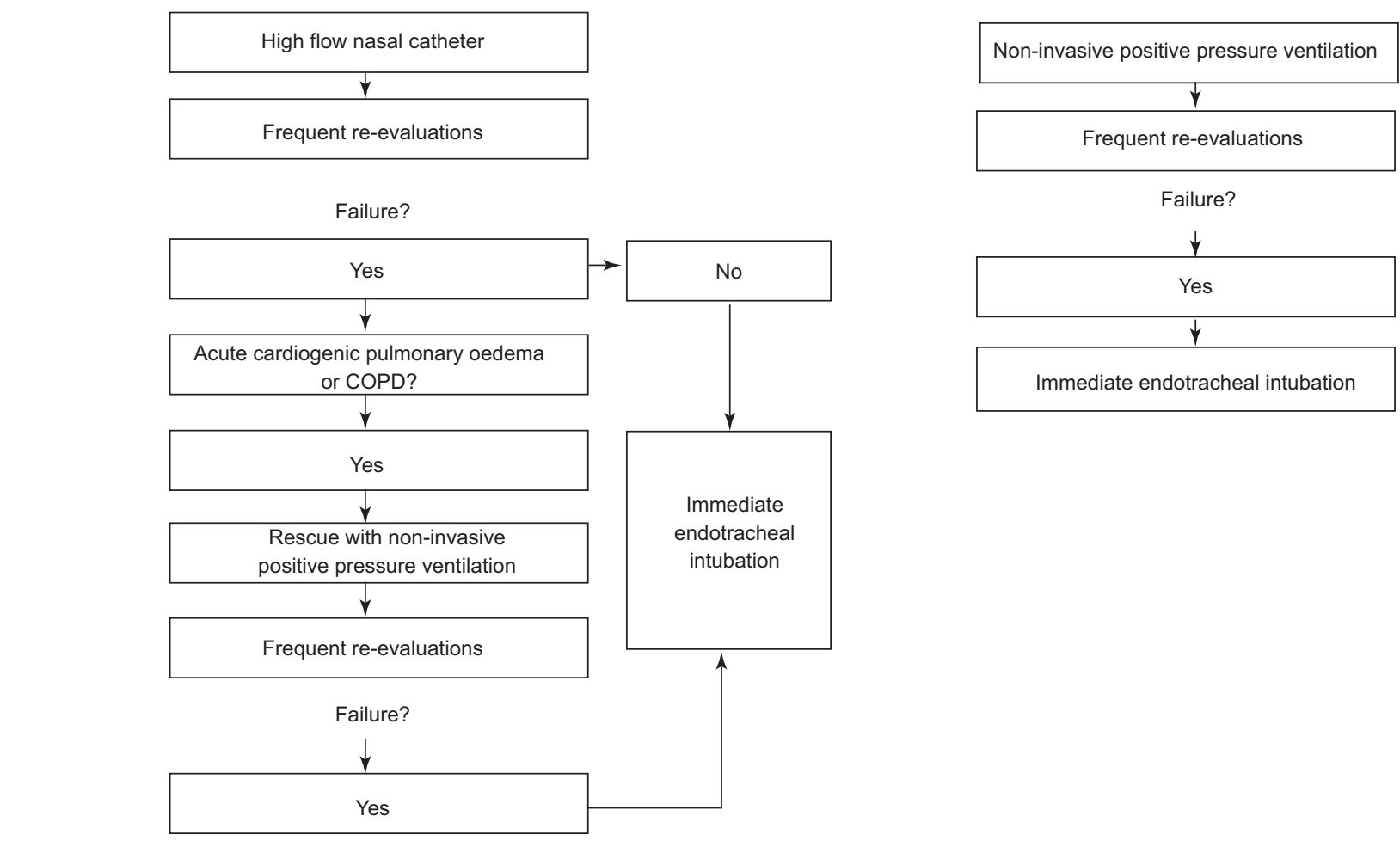

COPD = chronic obstructive pulmonary disease.

\section{Statistical analysis}

\section{Non-inferiority margin calculation}

RENOVATE is a non-inferiority Bayesian adaptive trial. To calculate the non-inferiority margin, we first considered the estimated absolute effect of NIPPV compared with low flow oxygen on intubation rate as $36 \% .{ }^{34}$ Considering that the non-inferiority margin should not be greater than half of this difference, ${ }^{35} 10 \%$ was chosen. This is equivalent to an odds ratio of 1.33 (a 10\% absolute margin corresponds to a relative variation of $33 \%$ assuming a control event rate of $40 \%$ ). Subsequently, that margin was converted into a logarithmic scale, generating a logarithm of the odds ratio (log odds) of 0.44 , which was used for simulations for power calculation.

\section{Simulations, sample size and power calculations}

The assumptions used in the non-inferiority margin calculation were evaluated under a variety of possible scenarios for the real underlying treatment effect in each group of interest. Hierarchical model, interim analysis schedule, stopping rules, and sample size and power calculations are discussed in the Online Appendix (sections
S1, S2, S3 and S4). Simulations were conducted to develop the adaptive rules and understand their performance ( $\mathbf{S 2}$, S4). The changes in prevalence of the different ARF groups in our patient population secondary to the coronavirus disease 2019 (COVID 19) pandemic were considered under a new set of simulations to assure power was maintained (S4).

\section{Primary endpoint}

Analysis of the primary endpoint uses sequential testing of nested hypotheses, first testing for non-inferiority (versus futility), then testing for superiority if non-inferiority is achieved. The sample size for the trial is capped but not fixed, allowing patient groups to stop accruing once sufficient information is obtained to reach conclusion. It will depend on information obtained from accrued data. For example, if accrued data do not show any promise of superiority, there is no need to recruit 2000 patients and the superiority hypothesis will not be tested.

We define $\theta \mathrm{g}$ as the log odds for the treatment in group of interest $\mathrm{g}$. For the superiority analysis, the null hypothesis $\left(\mathrm{H}_{0}\right)$ is set at $\theta g \geq 0$ and the alternative hypothesis $\left(H_{1}\right)$ is set at $\theta g<0$. The non-inferiority analysis considers $\theta \mathrm{g}<0.442\left(\mathrm{H}_{1}\right)$, meaning 


\section{Table 3. Suggested criteria for endotracheal intubation}

\section{- Respiratory or cardiac arrest}

- Haemodynamic instability with mean arterial pressure $<65 \mathrm{mmHg}$, systolic arterial pressure $<90 \mathrm{mmHg}$ after proper fluid resuscitation, or need for increasing doses of vasopressors (>0.3 $\mu \mathrm{g} / \mathrm{kg} / \mathrm{min})$

- Mental confusion and agitation that prevents medical/nursing care without full sedation

- Glasgow coma scale score $<11$

- Failure to maintain $\mathrm{Spo}_{2}>92 \%$ (or $>88 \%$ in patients with COPD) despite $\mathrm{FiO}_{2}$ of $60 \%$

- Progressive increase $>10 \mathrm{mmHg}$ in $\mathrm{PacO}_{2}$ and concurrent drop in arterial blood pH despite unsuccessful attempts of improving ventilation

- Inability to cooperate with and/or tolerate a high flow nasal catheter and/or NIPPV

- Development of hypersecretion and inability to eliminate such secretion, requiring frequent discontinuation of NIPPV therapy

- Severe arrhythmia with haemodynamic instability

- Persistent respiratory acidosis with arterial blood $\mathrm{pH}<7.2$ after 60 minutes of optimal treatment

- Endotracheal intubation at physician discretion

- Intubation should also be considered in patients with:

- a ROX index score $<2.85$ at any time of evaluation after the second hour of therapy (score is obtained by dividing $\mathrm{Spo}_{2} / \mathrm{FiO}_{2}$ ratio by respiratory rate); 32 or

- hypoxaemic acute respiratory failure that maintains $\mathrm{Pao}_{2} / \mathrm{Fio}_{2}$ ratio $<200$ and tidal volume $>9.5 \mathrm{~mL} / \mathrm{kg}$ in the first $4 \mathrm{~h}$ of NIPPV therapy 33

$\mathrm{COPD}=$ chronic obstructive pulmonary disease. $\mathrm{FiO}_{2}=$ fraction of inspired oxygen. $\mathrm{NIPPV}=$ non-invasive positive pressure ventilation. PacO ${ }_{2}=$ partial pressure of arterial carbon dioxide. $\mathrm{PaO}_{2}=$ partial pressure of arterial oxygen. $\mathrm{ROX}=$ respiratory rate-oxygenation. $\mathrm{Spo}_{2}=$ oxygen saturation measured by pulse oximetry.

that the treatment is no worse than control group up to a $\log$ odds of 0.442 . The $H_{0}$ for non-inferiority is $\theta g \geq 0.442$.

The Bayesian model utilises a hierarchical model for the treatment effect across the four patient groups, which intrinsically accounts for multiple comparisons. The final analysis will follow the intention-to-treat principle and will occur when full data are obtained. Superiority within the group of interest will be declared if the probability of $\theta \mathrm{g}<0$ is higher than 0.98. Non-inferiority will be declared if the probability of $\theta \mathrm{g}<0.442$ is higher than 0.98 . If neither of these conditions are met, then the conclusion for the group is futility. The trial allows for early stopping for each group of interest for three reasons: futility, success on superiority, or success on non-inferiority with low prospects of achieving superiority.

The trial will enrol up to 2000 patients, and there are no enrolment caps for individual patient groups - all patients are accepted for groups of interest that have not been stopped. The first interim analysis was conducted after complete data for the first 500 patients became available. After the first interim analysis, up to six subsequent interim analyses will be performed, with the trial continuing provided that at least one patient group has not been stopped by the early stopping rules. Each subsequent interim analysis is triggered when an additional $X_{i}$ patients have been enrolled, where the value of $X$ depends on whether the hypoxaemic ARF group has been stopped. Since about $70 \%$ of patients are expected to be in the hypoxaemic ARF group, $X_{i}$ is 250 if this group has not been stopped, and $X_{i}$ is 50 if it has been stopped. At each interim analysis, the groups of interest are evaluated for early stopping, provided that a minimum number of patients has been enrolled.

A hierarchical model will be fitted to provide posterior probabilities of the hypotheses for evaluating group of interest stopping. The hierarchical model allows for borrowing of information about treatment effect between groups. The stopping rules applied to each group of interest are discussed in the Online Appendix (section S1). The final analysis will occur after all groups have been stopped and complete data become available for any remaining enrolled patients.

\section{Secondary outcomes}

The 28-day mortality rate will use the same structure as the Bayesian model for the primary endpoint, with modification to prior distributions to reflect the differing rates for 28 day mortality, and with no consideration of a non-inferiority margin (Online Appendix, section S3).

For other secondary endpoints, inferences will be based on a Bayesian linear and logistic regression model that will calculate the posterior probability of superiority of the intervention considering data accumulated during the trial 


\section{ORIGINAL ARTICLES}

with uninformative prior distribution. Superiority will be declared if the probability that the intervention is superior to control is $>99 \%$. The definitions for the other secondary outcomes are explained in the Online Appendix (section S3).

\section{Exploratory analyses}

We will apply the Gail-Simon test to assess quantitative interactions between the study arms and the following prespecified subgroups for each group of ARF:

- body mass index $<35 \mathrm{~kg} / \mathrm{m}^{2}$ or $\geq 35 \mathrm{~kg} / \mathrm{m}^{2}$;

- time from randomisation to endotracheal intubation $<3$ days or $\geq 3$ days;

- NIPPV time before randomisation $<3 \mathrm{~h}$ or $\geq 3 \mathrm{~h}$;

- duration of respiratory treatment before randomisation $<$ median or $\geq$ median;

- $\mathrm{PaO}_{2} / \mathrm{FiO}_{2}$ ratio < 200 or $\geq 200$;

- Simplified Acute Physiology Score 3 (SAPS 3) < median or $\geq$ median; and

- male or female.

\section{Sensitivity analyses}

Two sensitivity analyses will be conducted - one perprotocol analysis, and one analysis in the COPD and ACPO groups using scaling up ventilatory support (from NIPPV to endotracheal intubation and from HFNC therapy to noninvasive positive pressure ventilation and/or endotracheal intubation) as the outcome. The per-protocol population is defined as those patients who were given their allocated respiratory support (HFNC therapy or NIPPV) for at least 8 hours a day for the first 24 hours without crossover between treatment groups.

\section{Adverse events}

We will collect data on suspected unexpected severe adverse events. Such events must be reported to the coordinating site within 24 hours.

\section{Protocol violations and deviations}

Major deviations relating to the inclusion criteria, exclusion criteria, study conduct and patient management must be reported to the coordinating site.

\section{Trial organisation and oversight}

The trial's steering committee has seven members (study investigators) and is responsible for developing the study protocol, drafting manuscripts and submitting manuscripts for publication. A team from the HCor Research Institute coordinates the study in association with the Brazilian Research in Intensive Care Network (BRICNet). The HCor
Research Institute is responsible for conducting the study and for managing and controlling data quality. This protocol manuscript follows SPIRIT guidelines ${ }^{36}$ (Online Appendix, section S5).

The data safety and monitoring board (DSMB) is composed of an external statistician and three researchers who are all experts in critical care medicine (see DSMB Charter in the Online Appendix, section S6). The DSMB is responsible for the interim analysis and for providing guidance to the steering committee regarding the continuation and safety of the trial after the interim analyses.

\section{Data collection and management}

Demographic and baseline data, height, SAPS 3 and history of comorbidities are collected for all patients. Gas exchange data, ventilatory parameters, and haemodynamic and laboratory data are collected before randomisation until Day 7. Data on mechanical ventilation use, other ventilation and oxygen support parameters, sedative use, vasopressor infusion and vital status are collected daily until Day 28 or until hospital discharge, whichever comes first.

All data are collected via an electronic case report form and periodical data quality checks are conducted. Database lock will be carried out after 90-day outcomes are obtained for all patients. Database access will be granted to only the steering committee members and statisticians before the main results are published. We plan to share the trial data with investigators who are conducting ongoing clinical trials on the same topic, for the purpose of using individual patient data in meta-analyses, and upload the study dataset to a public database.

\section{Current trial status}

Between November 2019 and June 2021, 736 patients were randomly assigned to one of the study arms. The first DSMB meeting was held in April 2021. After analysing data according to the DSMB Charter (Online Appendix, section S6), the DSMB recommended interrupting enrolment of the immunocompromised patients group due to futility, and this recommendation was implemented on 13 April 2021.

\section{Extenuating circumstances}

RENOVATE has had no major change in its protocol. However, the COVID-19 pandemic slowed recruitment at some sites due to work overload, pandemic foresight activities, and infection control precautions. The types of patients admitted to ICUs changed considerably, with a steep increase in COVID-19 hypoxaemic ARF patients and 


\section{ORIGINAL ARTICLES}

a decrease in all other groups of ARF admissions. Details regarding the impact of the pandemic on the study are in the Online Appendix (section S7).

\section{Ethical considerations and dissemination}

The trial was designed according to the guidelines for Good Clinical Practice and the principles of the Declaration of Helsinki. It was approved by the Brazil's national committee on ethics in research on 19 May 2021 (Comissão Nacional de Ética em Pesquisa [CONEP] n. 3.734.371; protocol version 5) and each participating centre's ethics committee. Patients are included before the informed consent form is obtained by the study's investigators due to the emergency of clinical decisions regarding respiratory support. Patients and/or their legal representatives are approached at the first opportunity after clinical stability is achieved. If patient dies before investigator's contact with legal representative, data usage permission is asked to centre ethics committee.

The study will be submitted for publication after completion irrespective of its findings. Manuscript preparation will be an inalienable responsibility of the steering committee. The main paper will be authored by the steering committee members plus the principal investigators of the top-ten recruiting sites which can contribute intellectually to the manuscript.

\section{Role of the sponsor source}

Brazil's Ministry of Health — through its PROADI-SUS (Programa de Apoio e Desenvolvimento Institucional do Sistema Unico de Saúde) - provides the grant funding for RENOVATE. All centres were provided with AIRVO 2 devices together with hands-on training by the Fisher and Paykel team. Neither the Brazilian Ministry of Health nor Fisher and Paykel are involved in the study design, data analysis, manuscript preparation or decision to submit the results for publication.

\section{Discussion}

The RENOVATE trial is a Bayesian adaptive study with a non-fixed sample size recruiting four subgroups of ARF - hypoxaemic non-immunocompromised, hypoxaemic immunocompromised, COPD exacerbation, and ACPO - to evaluate treatment effects in these subgroups simultaneously. The dynamic hierarchical borrowing model used allows information on the treatment effect in one subgroup of ARF to be borrowed when estimating the treatment effect in another subgroup. ${ }^{37}$ Hence, power can be increased to answer our research question in all groups in one trial at the same time, thereby saving time and resources.

This study has limitations. First, there is a risk of losing power in case of greater heterogeneity in some patient subgroups, as may be expected in the COPD and ACPO subgroups, which has been foreseen and properly adjusted for in the statistical analysis plan. Second, commencing the trial very close to the time of the COVID-19 pandemic onset has slowed our recruitment rates due to an enormous sudden increase in workload at the participating centres, and increased our hypoxaemic patient subpopulation at expense of other subpopulations. Third, the study protocol is being published several months after patient recruitment began. However, the content of this article is consistent with the protocol submitted to and approved by the national and local ethics committees and that registered at ClinicalTrials.gov.

In conclusion, RENOVATE is expected to answer important research questions regarding different ARF scenarios in only one trial via its a unique adaptive design, testing different hypotheses (superiority and non-inferiority) for patientcentred outcomes, which will save time and resources while assuring statistical power and patient safety.

Acknowledgements: This study is funded by the Brazilian Ministry of Health PROADI-SUS (Programa de Apoio e Desenvolvimento Institucional do Sistema Unico de Saúde). Fisher and Paykel provides high flow nasal catheter devices (AIRVO 2) but is not otherwise involved in the study design and analysis or the decision to submit the results.

\section{Competing interests}

All authors declare that they do not have any potential conflict of interest in relation to this manuscript.

Author details
Israel S Maia ${ }^{1,2}$
Leticia Kawano-Dourado $^{1,3}$
Fernando G Zampieri
Lucas P Damiani $^{1}$
Renato H Nakagawa ${ }^{1}$
Rodrigo M Gurgel ${ }^{1}$
Karina Negrelli
Samara PC Gomes ${ }^{1}$
Denise Paisani ${ }^{1}$
Lucas M Lima $^{1}$
Eliana V Santucci ${ }^{1}$
Nanci Valeis ${ }^{1}$
Ligia N Laranjeira $^{1}$
Roger Lewis,
Mark Fitzgerald
Carlos RR Carvalho




\section{ORIGINAL ARTICLES}

Laurent Brochard ${ }^{6,7}$

Alexandre B Cavalcanti ${ }^{1,2}$

For the RENOVATE Investigators and the BRICNet

1 HCor Research Institute, Hospital do Coracao, Sao Paulo, Brazil.

2 Anesthesiology Division, Medical School, University of Sao Paulo, Sao Paulo, Brazil.

3 Pulmonary Division, Medical School, University of Sao Paulo, Sao Paulo, Brazil.

4 University of California, Los Angeles (UCLA), Los Angeles, California, USA.

5 Berry Consultants, Austin, Texas, USA.

6 Keenan Research Centre for Biomedical Science, Li Ka Shing Knowledge Institute, St Michael's Hospital, Unity Health Toronto, Toronto, Canada.

7 Interdepartmental Division of Critical Care Medicine, University of Toronto, Toronto, Canada.

Correspondence: ismaia@ext.hcor.com.br

doi: https://doi.org/10.51893/2022.1.0A8

\section{References}

1 Franca SA, Toufen C Jr, Hovnanian AL, et al. The epidemiology of acute respiratory failure in hospitalized patients: a Brazilian prospective cohort study. J Crit Care 2011; 26: 330.e1-8.

2 Behrendt CE. Acute respiratory failure in the United States: incidence and 31-day survival. Chest 2000; 118: 1100-5.

3 Gattinoni L, Carlesso E, Langer T. Towards ultraprotective mechanical ventilation. Curr Opin Anaesthesiol 2012; 25: 1417.

4 Morandi A, Brummel NE, Ely EW. Sedation, delirium and mechanical ventilation: the 'ABCDE' approach. Curr Opin Crit Care 2011; 17: 43-9.

5 Muscedere J, Dodek P, Keenan S, et al. Comprehensive evidence-based clinical practice guidelines for ventilatorassociated pneumonia: diagnosis and treatment. J Crit Care 2008; 23: 138-47.

6 Safdar N, Dezfulian C, Collard HR, Saint S. Clinical and economic consequences of ventilator-associated pneumonia: a systematic review. Crit Care Med 2005; 33: 2184-93.

7 Drake MG. High-flow nasal cannula oxygen in adults: an evidence-based assessment. Ann Am Thorac Soc 2018; 15: 145-55.

8 Ritchie JE, Williams AB, Gerard C, Hockey H. Evaluation of a humidified nasal high-flow oxygen system, using oxygraphy, capnography and measurement of upper airway pressures. Anaesth Intensive Care 2011; 39: 1103-10.

9 Azevedo LC, Park M, Salluh Jl, et al. Clinical outcomes of patients requiring ventilatory support in Brazilian intensive care units: a multicenter, prospective, cohort study. Crit Care 2013; 17: R63.
10 Katz JA, Marks JD. Inspiratory work with and without continuous positive airway pressure in patients with acute respiratory failure. Anesthesiology 1985; 63: 598-607.

11 Fessler HE, Brower RG, Wise RA, Permutt S. Mechanism of reduced LV afterload by systolic and diastolic positive pleural pressure. J App/ Physiol (1985) 1988; 65: 1244-50.

12 Mauri T, Alban L, Turrini C, et al. Optimum support by high-flow nasal cannula in acute hypoxemic respiratory failure: effects of increasing flow rates. Intensive Care Med 2017; 43: 1453-63.

13 Mauri T, Turrini C, Eronia N, et al. Physiologic effects of highflow nasal cannula in acute hypoxemic respiratory failure. Am J Respir Crit Care Med 2017; 195: 1207-15.

14 Möller W, Feng S, Domanski U, et al. Nasal high flow reduces dead space. J App/ Physiol (1985) 2017; 122: 191-7.

15 Sztrymf B, Messika J, Bertrand F, et al. Beneficial effects of humidified high flow nasal oxygen in critical care patients: a prospective pilot study. Intensive Care Med 2011; 37: 1780-6.

16 Mauri T, Galazzi A, Binda F, et al. Impact of flow and temperature on patient comfort during respiratory support by high-flow nasal cannula. Crit Care 2018; 22: 120.

17 Frat JP, Thille AW, Mercat A, et al. High-flow oxygen through nasal cannula in acute hypoxemic respiratory failure. $N$ Engl J Med 2015; 372: 2185-96.

18 Bellani G, Laffey JG, Pham T, et al. Epidemiology, patterns of care, and mortality for patients with acute respiratory distress syndrome in intensive care units in 50 countries. JAMA 2016; 315: 788-800.

19 Ranieri VM, Rubenfeld GD, Thompson BT, et al; ARDS Definition Task Force. Acute respiratory distress syndrome: the Berlin definition. JAMA 2012; 307: 2526-33.

20 Macklem PT. Hyperinflation [editorial]. Am Rev Respir Dis 1984; 129: 1-2.

21 Juan G, Calverley P, Talamo C, et al. Effect of carbon dioxide on diaphragmatic function in human beings. N Engl J Med 1984; 310: 874-9.

22 Chadda K, Annane D, Hart N, et al. Cardiac and respiratory effects of continuous positive airway pressure and noninvasive ventilation in acute cardiac pulmonary edema. Crit Care Med 2002; 30: 2457-61.

23 Allison RC. Initial treatment of pulmonary edema: a physiological approach. Am J Med Sci 1991; 302: 385-91.

24 Ferreyro BL, Angriman F, Munshi L, et al. Association of noninvasive oxygenation strategies with all-cause mortality in adults with acute hypoxemic respiratory failure: a systematic review and meta-analysis. JAMA 2020; 324: 57-67.

25 Frat JP, Ragot S, Girault C, et al. Effect of non-invasive oxygenation strategies in immunocompromised patients with severe acute respiratory failure: a post-hoc analysis of a randomised trial. Lancet Respir Med 2016; 4: 646-52.

26 Azoulay E, Mokart D, Kouatchet A, et al. Acute respiratory failure in immunocompromised adults. Lancet Respir Med 2019; 7: 173-86.

27 Lemiale V, Resche-Rigon M, Mokart D, et al. High-flow nasal cannula oxygenation in immunocompromised patients with acute hypoxemic respiratory failure: a groupe de recherche 


\section{ORIGINAL ARTICLES}

respiratoire en réanimation onco-hématologique study. Crit Care Med 2017; 45: e274-80.

28 Yang PL, Yu JQ, Chen HB. High-flow nasal cannula for acute exacerbation of chronic obstructive pulmonary disease: a systematic review and meta-analysis. Heart Lung 2021; 50: 252-61.

29 Azoulay E, Lemiale V, Mokart D, et al. Effect of high-flow nasal oxygen vs standard oxygen on 28-day mortality in immunocompromised patients with acute respiratory failure: the HIGH randomized clinical trial. JAMA 2018; 320: 2099-107.

30 Rochwerg B, Brochard L, Elliott MW, et al. Official ERS/ATS clinical practice guidelines: noninvasive ventilation for acute respiratory failure. Eur Respir J 2017; 50: 1602426.

31 Nava S, Carbone G, DiBattista N, et al. Noninvasive ventilation in cardiogenic pulmonary edema: a multicenter randomized trial. Am J Respir Crit Care Med 2003; 168: 1432-7.

32 Roca O, Caralt B, Messika J, et al. An index combining respiratory rate and oxygenation to predict outcome of nasal high-flow therapy. Am J Resp Crit Care Med 2019; 199: 1368-76.

33 Carteaux G, Millán-Guillarte T, De Prost N, et al. Failure of noninvasive ventilation for de novo acute hypoxemic respiratory failure: role of tidal volume. Crit Care Med 2016; 44: 282-90.

34 Liu YJ, Zhao J, Tang H. Non-invasive ventilation in acute respiratory failure: a meta-analysis. Clin Med (Lond) 2016; 16: 514-23.

35 Jones B, Jarvis P, Lewis JA, Ebbutt AF. Trials to assess equivalence: the importance of rigorous methods. BMJ 1996; 313: 36-9.

36 Chan AW, Tetzlaff JM, Altman DG, et al. SPIRIT 2013 statement: defining standard protocol items for clinical trials. Ann Intern Med 2013; 158: 200-7.

37 Berry SM, Broglio KR, Groshen S, Berry DA. Bayesian hierarchical modeling of patient subpopulations: efficient designs of Phase II oncology clinical trials. Clin Trials 2013; 10: 720-34. 